\title{
ENSAIOS DE APRENDIZAGEM DE MÁQUINA NO BANCOS DE DADOS DO SISTEMA DE FISCALIZAÇÃO DE GESTÃ̃ DO TCE
}

\author{
Pablo Procópio Martins ${ }^{\mathbf{1}}$; \\ Edjandir Corrêa Costa ${ }^{2}$; \\ Aires José Rover ${ }^{3}$; \\ Paloma Maria Santos ${ }^{4}$;
}

\begin{abstract}
Motivated by the interest in making forecasts about the fulfillment of the Santa Catarina municipalities regarding the targets determined by the Fiscal Responsibility Law, this research, applied, quantitative and with an inductive approach, use data mining techniques and regression analysis to develop its object, which considered the net current revenue as a study variable and statistical comparison, using forecasting algorithms. The selected techniques showed that the executives' personnel expenses are close to $50 \%$ and that, among the algorithms analyzed, the best approach was obtained by RandomForest, which presented a relative absolute error of $5.1904 \%$. These results showed that it is feasible to predict whether a municipality will exceed the targets imposed by law, information that conditions the Audit Courts to act proactively, making inspection activities more effective.
\end{abstract}

Keywords: Electronic Government; Artificial intelligence; Data Mining; Machine Learning; Fiscal Responsibility Law;

Resumo: Motivada pelo interesse em realizar previsões acerca do cumprimento dos municípios catarinenses no que tange às metas determinadas pela Lei de Responsabilidade Fiscal, a presente pesquisa, de caráter aplicado, quantitativo e com abordagem indutiva, fez uso de técnicas de mineração de dados e análise de regressão para desenvolver seu objeto, que considerou a receita corrente líquida como variável de estudo e comparação estatística, com o emprego de algoritmos de previsão. As técnicas selecionadas evidenciaram que os gastos com pessoal dos executivos é próximo de $50 \%$ e que, dentre os algoritmos analisados, a melhor abordagem foi obtida pelo RandomForest, que apresentou um erro absoluto relativo de 5,1904\%. Tais resultados permitiram demonstrar que é viável prever se um município irá extrapolar as metas impostas pela lei, informação que condiciona os Tribunais de Contas a atuar de forma pró-ativa, tornando mais efetivas as atividades de fiscalização.

Palavras-chave: Governo Eletrônico; Inteligência Artificial; Mineração de Dados; Aprendizado de Máquina; Lei de Responsabilidade Fiscal;.

\footnotetext{
1 Programa de Pós-Graduação em Engenharia e Gestão do Conhecimento - Universidade Federal de Santa Catarina (UFSC) Florianópolis - Brasil. Correo electrónico: pablopmartins@gmail.com

2 Programa de Pós-Graduação em Engenharia e Gestão do Conhecimento - Universidade Federal de Santa Catarina (UFSC) Florianópolis - Brasil. Correo electrónico: edjandir@gmail.com

${ }^{3}$ Programa de Pós-Graduação em Engenharia e Gestão do Conhecimento - Universidade Federal de Santa Catarina (UFSC) Florianópolis - Brasil. Correo electrónico: aires.rover@ gmail.com

${ }^{4}$ Programa de Pós-Graduação em Engenharia e Gestão do Conhecimento - Universidade Federal de Santa Catarina (UFSC) Florianópolis - Brasil. Correo electrónico: santos.paloma@gmail.com
} 


\section{INTRODUÇÃO}

Ubiquidade tecnológica, sobrecarga de dados, diversidade de fontes de informação e escassez de conhecimento. Expressões que traduzem bem os tempos modernos. Cada vez mais conectadas, as organizações vêm lançando mão de estratégias que as permitam gerir suas bases de dados de maneira eficiente, tendo em vista utilizar o conhecimento pregresso para auxiliar no processo de tomada de decisão e na geração de novos conhecimentos.

No âmbito da justiça, técnicas de inteligência artificial e, em especial o aprendizado de máquina (Machine Learning - ML), vêm sendo testadas para dar vazão aos milhões de processos judiciais que atravancam o sistema judiciário como um todo. Por meio destas técnicas, busca-se, além da celeridade, alcançar uma maior segurança jurídica, previsibilidade e coerência nas decisões (Sabo \& Rover, 2020).

A aplicação desses mecanismos tecnológicos também pode ser vislumbrada no contexto da auditoria externa das contas públicas dos municípios. Com o apoio da inteligência artificial, fraudes podem ser auferidas, processos de fiscalização podem ganhar celeridade e até mesmo mais pró-atividade pode ser esperada dos apontamentos aos prefeitos.

A literatura científica aponta para um eminente debate sobre a introdução de práticas de inteligência artificial com o enfoque na extração de conhecimento a partir das bases de dados, mais precisamente a técnica de ML, para tornar os trabalhos mais céleres e efetivos. Apesar de os estudos se desenvolverem, em sua maioria, no Poder Executivo, projetos relevantes, como é o caso do VICTOR ${ }^{5}$, que executa uma primeira triagem dos processos que chegam ao Supremo Tribunal Federal, também são encontrados no Judiciário.

Dentro do escopo do Poder Legislativo e dos órgãos de controle, um exemplo de portal de governo eletrônico, com grande volume de dados que podem ser explorados para gerar conhecimento e novos bens e serviços a sociedade, é o do Tribunal de Contas de Santa Catarina (TCE/SC). Ele alimenta um banco de dados com informações relativas aos gastos municipais

\footnotetext{
${ }^{5} \mathrm{O}$ nome do projeto de ML do Supremo Tribunal Federal em conjunto com o grupo de pesquisa DR.IA da UnB Universidade de Brasília é VICTOR, em homenagem ex-Ministro deste tribunal, de 1960 a 1969, Victor Nunes Leal. O projeto tem o intuito de reconhecer padrões para classificar peças jurídicas (com textos e imagens) por meio de técnicas de ML para realizar classificação de temas de repercussão geral (Peixoto \& Dezan, 2020).
} 
do executivo e do legislativo, além das metas da Lei de Responsabilidade Fiscal (LRF) e outros gastos com saúde e educação, cujas receitas são auferidas por esses entes do Estado.

Além de diferentes caraterísticas, perfis demográficos e populacionais e necessidades de recursos para atender às demandas da sociedade e as de investimento público, distinto também é o desempenho econômico-financeiro dos municípios catarinenses. Este último fator, especificamente, foi apontado por Rodrigues, Schenitzler, Sallum \& Raupp (2019) como excessivamente fragmentado.

Com o propósito de verificar empiricamente tal observação, o presente estudo fez uso de técnicas de mineração de dados e análises de regressão junto aos dados financeiros e orçamentários dos municípios da grande Florianópolis, no intento de realizar previsões acerca do cumprimento das metas determinadas pela LRF.

A principal contribuição deste estudo é oferecer aos operadores de controle externo dos municípios catarinenses novas possibilidades para execução deste trabalho de forma mais próativa com as predições que a ferramenta ML oferece.

\section{REFERENCIAL TEÓRICO}

Essa seção irá lastrear teoricamente o experimento a ser apresentado repassando uma visão sobre a fiscalização dos municípios, a LRF, as ações do TCE/SC na automação de suas auditorias externas e as técnicas de inteligência artificial que propiciam a extração de conhecimento de dados.

\subsection{CONTROLE DA ADMINISTRAÇÃO PÚBLICA}

Uma das peculiaridades da administração pública, que a difere dos entes do setor privado, reside na função controle. Mesmo sem um consenso, ela é subdividida em controle externo, controle interno, controle social, controle judicial, controle administrativo e controle gerencial (Platt Neto, 2019).

Compete ao TCE, órgão de controle externo, nos termos da Constituição do Estado, da Legislação Orgânica e sua Resolução nº TC-06-2001, fiscalizar, inspecionar, analisar e auditar as prestações de contas das entidades que fizerem uso de recursos públicos do Estado de Santa 
Catarina, cuja análise envolve o cumprimento da Lei Complementar 101/2000, conhecida como LRF.

Conforme Artigos $1^{\circ}$ e 50 da Lei Orgânica do TCE/SC, compete ao tribunal:

$[\ldots]$

II - apreciar as contas prestadas anualmente pelo Prefeito Municipal, nos termos do art. 50 e seguintes desta Lei.

[...]

Art. 50. O Tribunal de Contas do Estado apreciará as contas prestadas anualmente pelo Prefeito, as quais serão anexadas às do Poder Legislativo, mediante parecer prévio a ser elaborado antes do encerramento do exercício em que foram prestadas (Tribunal de Contas de Santa Catarina, 2000).

Ao impor gastos dos entes federativos contrapondo suas capacidades arrecadatórias tributárias (Brasil, 2000), a LRF, agora tratada como um princípio de gestão, vislumbrou a possibilidade de obter transparência das ações governamentais em todos os âmbitos. Nos termos do Artigo 48 da referida lei,

[...] são instrumentos de transparência da gestão fiscal, aos quais será dada ampla divulgação, inclusive em meios eletrônicos de acesso público: os planos, orçamentos e leis de diretrizes orçamentárias; as prestações de contas e o respectivo parecer prévio; o Relatório Resumido da Execução Orçamentária e o Relatório de Gestão Fiscal; e as versões simplificadas desses documentos (Brasil, 2000).

Conforme Artigo 53, devem acompanhar a versão simplificada do relatório de execução orçamentária os demonstrativos relativos a apuração da RCL, as receitas e despesas previdenciárias, resultados nominal e primário, despesas com juros, e restos a pagar (Brasil, 2000).

Com a LRF os cidadãos são municiados de informações relativas as atividades financeiras do Estado, de forma clara e prévia (Cruz \& Afonso, 2018).

\subsubsection{Ações do TCE/SC na automação de suas auditorias externas}

Ao longo dos últimos anos, o TCE/SC vem desenvolvendo ações para automatizar os processos de auditoria em conjunto com as entidades auditadas, dado o constante aumento do volume de inspeções e fiscalizações a serem realizadas. 
Desde 2011, as entidades podem enviar os dados diretamente para os bancos de dados do TCE/SC por meio de uma plataforma web service, onde são analisados. Um painel de Business Intelligence construído pelo Núcleo de Informações Estratégicas - NIF/TCE-SC para facilitar tomada de decisão permite decidir o quê auditar e qual unidade auditar.

Com o intuito avançar e de tornar mais eficientes seus processos de fiscalização e auditoria externa das contas públicas dos Municípios do Estado de Santa Catarina, uma nova frente foi adotada: firmar um acordo de cooperação técnica com a Universidade do Estado de Santa Catarina com o intuito de desenvolver modelos de ML para auxiliar e tornar mais efetivas as atividades de fiscalização dos membros do Tribunal. Tal acordo viabilizou a pesquisa relatada no presente artigo.

\subsubsection{Extração de conhecimento em Dados}

A Inteligência Artificial, segundo Rover (2001), pode ser entendida como a simulação da inteligência humana exibida pelas máquinas, dada a maior capacidade que ganharam com o decorrer dos anos. Além da capacidade de aprender, os sistemas computacionais passaram a criar suas próprias heurísticas para solucionar problemas (Peixoto \& Dezan, 2020), reconhecer padrões e fazer inferências.

Ferramentas e técnicas que permitem explorar um grande volume de dados, identificar padrões compreensíveis e válidos e auxiliar no processo de descoberta de conhecimento, ganharam visibilidade e vêm sendo cada vez mais valorizadas e aplicadas no contexto das organizações.

A descoberta de conhecimento em base de dados (do inglês, KDD) é uma subcategoria do ML e tem como principal preocupação o encontro de conhecimento útil em um grande conjunto de dados. O KDD e o ML compartilham técnicas e algoritmos (Fawcett \& Provost, 2018), sendo que a grande diferença reside na sua finalidade. O ML busca a melhoria de performance e para isso conta com auxílio de outras disciplinas, como, por exemplo, a automação.

Os tomadores de decisão nas organizações precisam que estes dados sejam resumidos e organizados adequadamente para que, desta forma, sejam evidenciados padrões, relações e tendências. Essa descoberta pode ocorrer em três tipos de dados diferentes: os estruturados, que estão organizados na forma de tabela; os semiestruturados; e os não estruturados, em que a 
descoberta de conhecimento ocorre geralmente em fontes textuais (Fayyad, Piatetsky-Shapiro, Smyth, 1996).

Para que sejam encontradas as relações existentes entre os dados obtidos, diversos algoritmos podem ser utilizados. Um deles, orientado a encontrar um modelo de decisão para representar a relação entre os atributos, tem como objetivo o algoritmo máquina de vetores de suporte para regressão (SVR), e pode ser caracterizado como um algoritmo com aprendizado supervisionado (Vasconcelos, 2017).

No software de aplicação de aprendizagem de máquina, WEKA, o SVR é representado pela sigla SMOreg. Ele implementa um algoritmo sequencial de otimização mínima para aprender um modelo de regressão de vetores de suporte (Amaral, 2016).

A essência do SVR é achar um modelo de decisão que permita encontrar uma função que aproxime os pontos de treinamento com a meta de minimizar o erro de previsão por meio da minimização do limite superior da generalização do erro, sendo efetuada a soma do erro de treinamento e um nível de confiança (Vasconcelos, 2017).

\section{MÉTODOS DA PESQUISA}

O experimento relatado no presente artigo caracteriza-se como estudo de caso (Yin, 2015) por buscar investigar um fenômeno contemporâneo em seu contexto real. Foi empregado o método de abordagem indutiva (Lakatos \& Marconi, 2007) com a aplicação de análise estatística e técnicas de ML a fim de alcançar significados aos dados utilizados (Gil, 2008).

O estado de Santa Catarina foi escolhido como corpus desta pesquisa por conta da quantidade e diversidade de municípios que o compõe, quando comparado a sua dimensão territorial e populacional. Acredita-se que essa heterogeneidade pode apontar disparidades na hora de cumprir as condições impostas pela LRF.

A base de dados, cedida pelo TCE/SC, envolveu registros dos itens que compuseram os relatórios da LRF no período de 2015 a 2018, sendo a RCL eleita como variável de estudo e comparação estatística por representar o principal indicador para se auferir se despesas com pessoal estão dentro dos limites legais. O acesso a esses dados é fruto de um Acordo de Cooperação Técnica do referido tribunal com UFSC para aplicação de técnicas de ML para tornar os processos de fiscalização mais efetivos e pró ativos. 
O pré-processamento dos dados envolveu os seguintes passos: transposição dos indicadores e disposição em colunas; retirada dos estilos de formatação para permanecer apenas os dados brutos com formatação decimal; e exportação dos dados para o formato CSV para, futuramente, ser importado na ferramenta de data mining.

As análises foram intencionalmente direcionadas aos municípios da mesorregião da grande Florianópolis, cujos dados demográficos representam uma abrangência significativa. A amostra foi composta por três microrregiões (3) e um total de vinte e um municípios (21). As categorias de análise da matriz somaram um total de vinte e sete (27) indicadores, sendo alguns destes: Receita Corrente Líquida; Metas Bimestral de Arrecadação; valores líquidos de impostos IPTU, ISS, ITBI, Taxas e Contribuições de Melhoria; entre outros.

Além da planilha eletrônica, foi utilizado como suporte à análise o software WEKA 3.8, que permite testes e comparações com diversos algoritmos de ML e possibilita que se utilize abordagens diferentes, incluindo algoritmos estatísticos, abordagens baseadas em regras e redes neurais (Hall, Frank, Holmes, Pfahringer, Reutemann \& Witten, 2009).

Além da facilidade de uso e instalação, o software WEKA permite que sejam identificados e selecionados os atributos mais relevantes para construir predições, sendo esta uma das ações de suma importância quando se pretende minerar dados (Hall et al, 2009).

Assim, foi realizada uma combinação de diferentes métodos de pesquisa com critérios de avaliação auferindo a melhor técnica a ser empregada. A robustez do conjunto de atributos selecionado pode ser validada por meio de uma abordagem baseada em validação cruzada (Hall et al, 2009).

Após uma análise estatística dos dados, buscou-se identificar possíveis relações entre os indicadores municipais, a fim de, a partir de uma correlação, construir um modelo preditivo que possa ser utilizado em processos de governança e auditoria.

Duas correlações foram definidas: a primeira busca prever a RCL a partir dos primeiros resultados bimestrais e a segunda prevê o percentual de gastos dos poderes executivos e legislativo considerando um valor de RCL arbitrário.

Diferentes algoritmos de regressão (SimpleLinearRegression, SMOReg, Multilayer Perceptron, IBK, REPTree e Decision Table) foram executados para cada correlação com o propósito de avaliar o seu desempenho, tendo sido mantidos os parâmetros originais de configuração. De forma automática e aleatória, o software dividiu a base em dois grupos: o 
grupo de treinamento, que correspondeu a $80 \%$ do total de instâncias, e a base de testes, com os $20 \%$ restantes. A lista de predições assim como os indicadores de desempenho foram utilizados para avaliar comparativamente os algoritmos.

\section{RESULTADOS E DISCUSSÕES}

Uma análise estatística básica antecedeu as sessões de regressão linear. O propósito da atividade foi direcionar as investigações de busca de correlações e, também, obter um entendimento de como as metas correspondem às receitas municipais consolidadas. A Figura 1 mostra como essa correspondência se deu nos municípios de Biguaçu e Joinville no ano de 2015.

Figura 1 - Testes iniciais de correspondência entre as receitas líquidas bimestrais de 2015
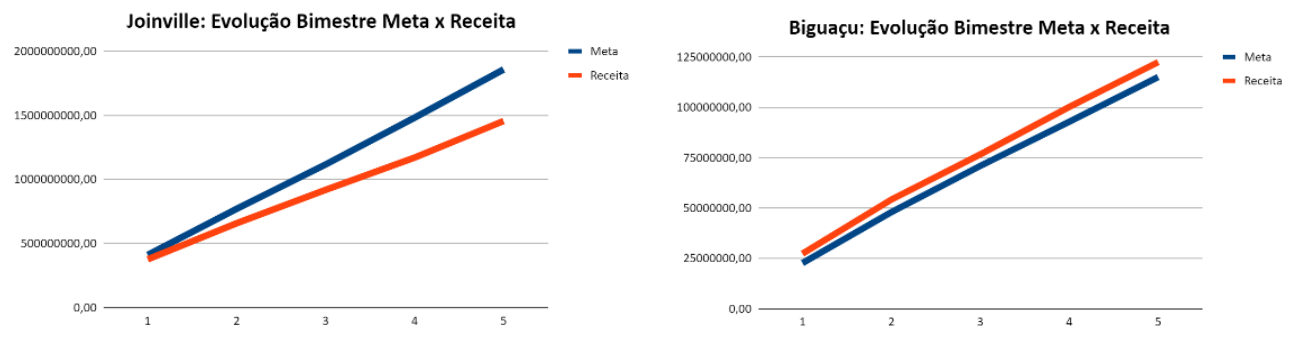

Fonte: Autores (2020)

Analisando os gráficos, evidencia-se que a linha que representa a RCL se afasta da linha que representa a meta de receita no município de Joinville a partir do segundo bimestre de 2015 . O mesmo fato não acontece no município de Biguaçu. Para se obter um entendimento sobre o que motivou a discrepância seria necessário investigar também fatores como os diferentes tipos de arrecadação e repasses federal e estadual esperados porém não realizados. Contudo, essa tarefa está fora do escopo deste trabalho.

A evolução do percentual de gastos municipais em relação à RCL também foi alvo de investigação. Os dados apresentados na Figura 2 foram obtidos no Portal do TCE/SC 6 . A série

\footnotetext{
${ }^{6}$ http://servicos.tce.sc.gov.br/sic/homesic.php
} 
inclui o percentual de gastos com os poderes executivo e legislativo dos municípios de Biguaçu, Florianópolis, São José e Palhoça nos últimos dez anos.

Figura 2 - Testes iniciais com o percentual de gastos e as receitas líquidas de 2008 a 2018

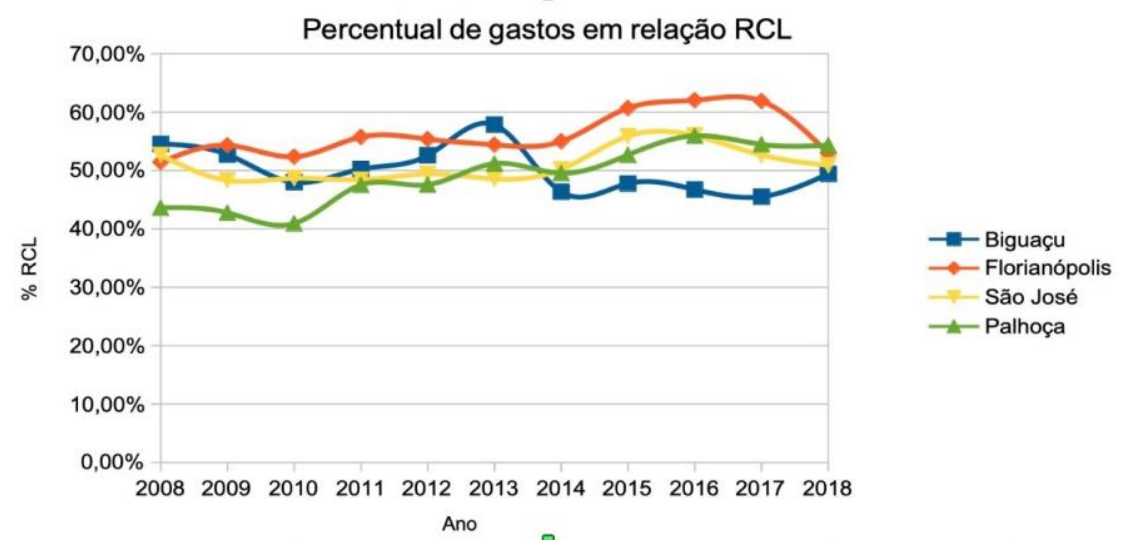

Fonte: Autores (2020)

A sobreposição de séries facilitou a comparação entre os municípios e mostrou que o percentual de gastos com pessoal do executivo oscila na faixa dos $50 \%$ em todos eles. $\mathrm{O}$ município de Florianópolis excedeu o limite proposto pela LRF nos anos de 2015, 2016 e 2017. Os fatores que levam a um aumento considerável de gastos devem ser avaliados individual e conjuntamente para se buscar um entendimento sobre tal fenômeno em experimentos que compare outras variáveis além das contidas neste estudo.

A primeira experiência realizada no software WEKA foi a tarefa de regressão linear que buscou estabelecer um modelo para previsão da RCL com base no resultado dos primeiros e segundos bimestres de cada município. Os dados exportados no formato CSV foram convertidos para o formato ARRF, proprietário da ferramenta. Foram selecionadas três características das 32 presentes em cada instância (Figura 3). As demais características foram removidas da sessão.

Figura 3 - Experimentos com regressão multivariada, atributos - rcl; receita1; receita2 


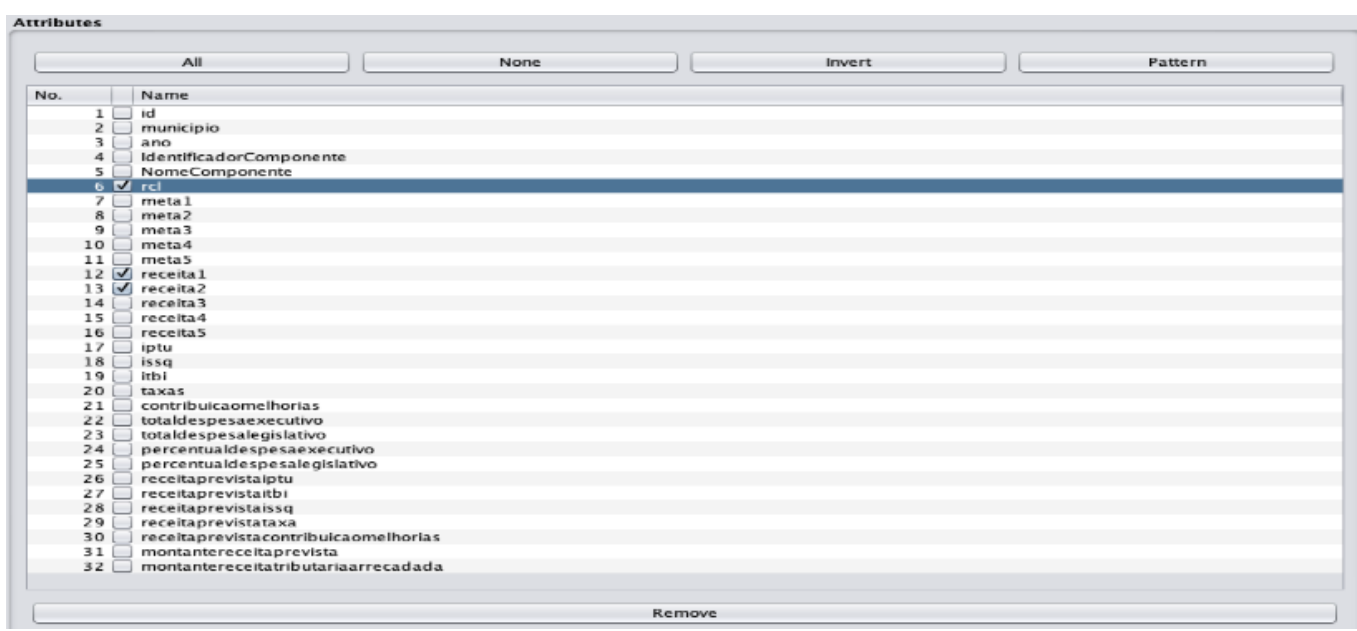

Fonte: Autores (2020)

As variáveis independentes receita1 e receita2, que representam respectivamente a receita apurada nos primeiros e segundos bimestres do ano, são utilizadas para a construção da função que busca prever a RCL anual.

A Tabela 1 mostra o resultado da execução da regressão linear para cada algoritmo classificador. A ferramenta WEKA mostra como saída do processo todas as previsões e um resumo com os valores dos indicadores de desempenho do algoritmo. Os indicadores foram obtidos considerando as previsões de teste que, nesse experimento, correspondeu a $20 \%$ do total de 180 instâncias.

Tabela 1- Resultado da execução da regressão linear para cada algoritmo

\begin{tabular}{|l|c|c|c|}
\hline \multicolumn{1}{c|}{ Classificador } & $\begin{array}{c}\text { Coeficiente de } \\
\text { correlação }\end{array}$ & $\begin{array}{c}\text { Erro absoluto } \\
\text { relativo }\end{array}$ & $\begin{array}{c}\text { Raiz quadrada de erro } \\
\text { relativo }\end{array}$ \\
\hline SimpleLinearRegression & 0,9991 & $4,2923 \%$ & $4,4565 \%$ \\
\hline SMOreg & 0,9988 & $4,1933 \%$ & $5,1114 \%$ \\
\hline IBK & 0,9994 & $5,0065 \%$ & $6,2553 \%$ \\
\hline AlternateModelTree & 0,9983 & 4,2658 & $5,9258 \%$ \\
\hline MultilayerPerceptron & 0,9993 & $3,2174 \%$ & $5,3185 \%$ \\
\hline
\end{tabular}

Fonte: Autores (2020) 
A ferramenta mostrou índices de desempenho semelhantes dos algoritmos classificadores na tarefa de regressão realizada. Foi possível alcançar um resultado aceitável para as previsões, considerando que o erro absoluto médio ficou, no geral, em torno de 4\%. Das opções avaliadas, a que teve o melhor desempenho foi a abordagem de redes neurais, representada neste experimento pela implementação MultilayerPerceptron.

A segunda experiência realizada foi a construção de uma função que faz a previsão do percentual de gastos com base em uma RCL arbitrária. A base foi formada por 132 instâncias, e, para cada uma, foram selecionadas duas variáveis. Este modelo pode ser útil para prever gastos baseados em um histórico disponível. O desempenho dos algoritmos classificadores nesta experiência é demonstrado na Tabela 2.

Tabela 2- Desempenho dos algoritmos classificadores

\begin{tabular}{|l|c|c|c|}
\hline \multicolumn{1}{c|}{ Classificador } & $\begin{array}{c}\text { Coeficiente de } \\
\text { correlação }\end{array}$ & $\begin{array}{c}\text { Erro absoluto } \\
\text { relativo }\end{array}$ & $\begin{array}{c}\text { Raiz quadrada de erro } \\
\text { relativo }\end{array}$ \\
\hline SimpleLinearRegression & 0,995 & $8,0998 \%$ & $10,6209 \%$ \\
\hline SMOreg & 0,995 & $7,8048 \%$ & $11,2228 \%$ \\
\hline IBK & 0,9975 & $5,9144 \%$ & $7,8403 \%$ \\
\hline RandomForest & 0,9982 & $5,1904 \%$ & $6,7659 \%$ \\
\hline MultilayerPerceptron & 0,9966 & $8,7592 \%$ & $9,3385 \%$ \\
\hline
\end{tabular}

Fonte: Autores (2020)

Na segunda experiência os resultados de desempenho mantiveram-se próximos. Porém, neste cenário, a opção mais efetiva foi a implementação RandomForest, que apresentou o menor valor de erro absoluto relativo. As duas experiências mostraram que é possível realizar previsões utilizando a técnica de regressão linear.

\section{CONCLUSÕES}


Motivado pelo interesse em realizar previsões acerca do cumprimento dos municípios catarinenses no que tange às metas determinadas pela LRF, o presente estudo fez uso de técnicas de mineração de dados aliado a análises de regressão junto aos dados financeiros e orçamentários fornecidos pelo TCE/SC.

O tratamento dos dados e a análise comparativa realizada entre os municípios da grande Florianópolis evidenciou que os gastos com pessoal dos executivos é próximo de 50\%, o limite máximo da LRF, sendo que a capital extrapolou essa margem nos anos de 2015 a 2017.

A partir da aplicação dos algoritmos de ML no software WEKA, chegou-se ao resultado que a melhor abordagem foi o RandomForest, que apresentou um erro absoluto relativo de $5,1904 \%$. Nos demais critérios, raiz quadrada de erro relativo e o coeficiente de correlação, esse algoritmo mostrou um desempenho também melhor que os demais, atingindo $6,7659 \%$ e 0,9982, respectivamente,

Esse experimento demonstrou que é possível, com a aplicação de técnicas de aprendizagem de máquina, realizar previsões sobre o comportamento de um município no que diz respeito aos limites estabelecidos pela LRF. Os resultados apresentados permitem que os Tribunais de Contas atuem de forma pró-ativa, tornando mais efetivas as atividades de fiscalização. Vale ressaltar, contudo, que previsões com pouca acurácia podem levar a ações desnecessárias por parte dos Tribunais, o que pode ser custoso ou conflitante perante outros atores da sociedade.

Como oportunidades de estudos futuros envolvendo técnicas de aprendizagem de máquina e os dados de um Tribunal de Contas, podem ser citadas: investigar a relação entre os dados da LRF e o real investimento dos municípios em serviços públicos de saúde ou educação; a aferição de possíveis conluios em dados de licitações de municipais utilizando as mesmas técnicas de clusterização; e a análise de outras variáveis além daquelas que foram utilizadas neste estudo.

\subsection{AGRADECIMENTOS}

O presente trabalho foi realizado com apoio do Governo eletrônico, inclusão digital e sociedade do conhecimento (UFSC), com linha de pesquisa e-Gov no Poder Judiciário, poio da Coordenação de Aperfeiçoamento de Pessoal de Nível Superior - Brasil (CAPES) - Código de Financiamento 001 e incentivo institucional da Universidade do Estado de Santa Catarina 
(UDESC) e Instituto Federal de Santa Catarina (IFSC). Cabe o agradecimento ao Tribunal de

Contas de Santa Catarina - TCE/SC que através do acordo de cooperação técnica forneceu os dados para realização deste experimento.

\section{REFERÊNCIAS}

Amaral, F. (2016). Aprenda mineração de dados: teoria e prática (Vol. 1). Alta Books Editora.

Brasil, S. F. do (1988). Constituição da república federativa do Brasil. Brasília: Senado Federal, Centro Gráfico.

Brasil, C., \& da União, C. G. (2000). Lei Complementar $n^{o}$ 101, de 4 de maio de 2000. Estabelece normas de finanças públicas voltadas para a responsabilidade na gestão físcal e dá outras providências. Brasília, DF: Senado.

Cruz, C. F. D., \& Afonso, L. E. (2018). Gestão fiscal e pilares da Lei de Responsabilidade Fiscal: evidências em grandes municípios. Revista de Administração Pública, 52(1), 126-148. Recuperado de https://www.scielo.br/pdf/rap/v52n1/1982-3134-rap-52-01126.pdf

Fayyad, U., Piatetsky-Shapiro, G., \& Smyth, P. (1996). From data mining to knowledge discovery in databases. AI magazine, 17(3), 37-37. Recuperado de https://www.aaai.org/ojs/index.php/aimagazine/article/view/1230

Fawcett, T., \& Provost, F. (2018). Data Science para Negócios: O que você precisa saber sobre mineração de dados e pensamento analítico de dados. Alta Books Editora.

Gil, A. C. (2008). Métodos e técnicas de pesquisa social. 6. ed. Ediitora Atlas SA.

Hall, M., Frank, E., Holmes, G., Pfahringer, B., Reutemann, P., \& Witten, I. H. (2009). The WEKA data mining software: an update. ACM SIGKDD explorations newsletter, 11(1), 10-18. Recuperado em https://www.kdd.org/exploration_files/p2V11n1.pdf

Yin, R. K. (2015). Estudo de Caso-: Planejamento e métodos. Bookman editora.

Lakatos, E. M., \& Marconi, M. D. A. (2007). Fundamentos de metodologia científica. 5. reimp. São Paulo: Atlas, 310.

Peixoto, F. H. \& Dezan, M. L. (2020) Soluções de inteligência artificial como forma de ampliar a segurança jurídica das decisões jurídicas. Revista Democracia Digital e Governo Eletrônico, v. 1 n 18 Ed Especial Direito e IA, Florianópolis. p. 178-190. Recuperado de http://buscalegis.ufsc.br/revistas/index.php/observatoriodoegov/article/view/325

Platt Neto, O. A. (2019). Contabilidade Pública: atualizada e focada. Universidade Federal de Santa Catarina (UFSC), 22. ed. rev. e atual. Florianópolis: Edição do autor.

Rodrigues, A. F. O., Schenitzler, E. L., Sallum, S. B., \& Raupp, F. M (2019). Fragmentação de municípios catarinenses e desempenho econômico-financeiro. Revista Pensamento Contemporâneo em Administração, 13(3), 126-142. Recuperado de https://periodicos.uff.br/pca/article/view/28270/pdf 
Rover, A. J. (2001). Informática no direito: inteligência artificial: introdução aos sistemas especialistas legais. Juruá Editora.

Sabo, I. C., \& Rover, A. J. (2020). Observância De Precedentes E Gestão De Demandas Repetitivas por meio do Aprendizado De Máquina. Revista Opinião Jurídica $18(28)$, 69-93.

Recuperado de https://periodicos.unichristus.edu.br/opiniaojuridica/article/view/2553/1104

Tribunal de Contas do Estado de Santa Catarina. (2000). Lei Complementar $n^{o} 202$, de 15 de dezembro de 2000. Institui a Lei Orgânica do Tribunal de Contas do Estado de Santa Catarina e adota outras providências. Florianópolis, SC.

Vasconcelos, L. P. (27 a 30 de agosto de 2017). Uma comparação de técnicas de regressão para a previsão de consumo de energia residencial no cenário nacional. XLIX Simpósio Brasileiro de Pesquisa Operacional. Blumenau. Recuperado de http://www.sbpo2017.iltc.br/pdf/169301.pdf 